\title{
Scalar PDF and velocity-scalar PDF modelling of the bluff-body stabilised flame HM1 using ILDM
}

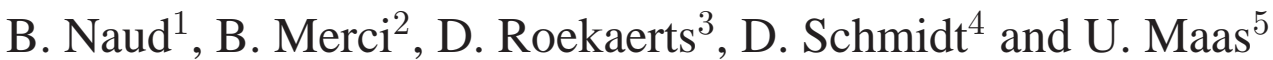 \\ ${ }^{1}$ Modeling and Numerical Simulation Group, Energy Dept., Ciemat, \\ Avda. Complutense 22, 28040 Madrid, Spain, bertrand.naud@ciemat.es \\ ${ }^{2}$ Dept. of Flow, Heat and Combustion Mechanics, Ghent University - UGent, \\ B-9000 Ghent, Belgium Bart.Merci@UGent.be \\ ${ }^{3}$ Department of Multi-Scale Physics, Delft University of Technology, \\ Lorentzweg 1, 2628 CJ Delft, The Netherlands,d.j.e.m.roekaerts@tudelft.nl \\ ${ }^{4}$ Institute for Internal Combustion Engines and Automotive Engineering, University of Stuttgart, \\ Pfaffenwaldring 12, D-70569 Stuttgart, Germany, dschmidt@itv.uni-stuttgart.de, \\ ${ }^{5}$ Institute for Technical Thermodynamics, Karlsruhe University (TH), \\ Kaiserstraße 12, 76131 Karlsruhe, Germany, umaas@itt.uni-karlsruhe.de
}

\begin{abstract}
Velocity-scalar and scalar PDF results are compared for the bluff-body stabilised flame $\mathrm{HM} 1$ using ILDM chemistry based on mixture fraction, and $\mathrm{CO}_{2}$ and $\mathrm{H}_{2} \mathrm{O}$ mass fractions. The same Reynolds stress turbulence model and the same modified Curl mixing model are used. No effect of radiative heat loss is included. The results for mean velocity and Reynolds stresses are satisfactory and very similar for both calculations. Each PDF modelling approach implies a different closure for the velocity-scalar correlation. In the present calculations this leads to significant differences in the radial profiles of mean scalars and of mixture fraction variance (different scalar flux modelling): velocity-scalar PDF results (differential scalar flux model) are better than scalar PDF results (gradient diffusion). Results in composition space (scatter plots) confirm the higher quality of the velocity-scalar PDF.
\end{abstract}

\section{Introduction}

Non-linear interaction between turbulent fluctuations and finite-rate chemistry can play an important role in nonpremixed turbulent flames, leading for instance to local extinction or incomplete combustion. Turbulence-chemistry interaction is therefore a central issue in nonpremixed turbulent flame modelling. Considering the modelling at the level of the joint scalar probability density function (PDF) offers an exact treatment of the chemical reaction source term (given a chemistry model).

In transported PDF methods based on stochastic Lagrangian modelling [1], a transport equation for the mass density function (MDF) is modelled and solved using a particle stochastic method. Three main modelling ingredients are required: turbulence model, chemistry model and micromixing model. Recently, several comparative studies have focussed on the influence of those different ingredients. In [2], three widely used mixing models are compared by considering stochastic simulations of partially stirred reactors (PaSR). In the context of transported scalar PDF modelling, the same mixing models are compared in [3] for the piloted jet diffusion flame Delft flame III, and in [4] for the bluff-body stabilised flames HM1-3. In [5], seven chemical mechanisms for methane are compared for joint velocity-scalar-turbulence frequency PDF 
calculations of the nonpremixed piloted jet flames D, E, and F. In [6], the influence of three $\mathrm{C}_{1}$ chemistry models on two mixing models is studied for Delft flame III and the bluff-body flames HM1-3.

Next to those three modelling ingredients, the choice of the PDF description itself has direct consequences on the modelling of the scalar flux (and higher order velocity-scalar correlation). When considering the joint scalar MDF $\mathcal{F}_{\phi}$, the use of a gradient diffusion assumption to close the conditional fluctuating velocity term in the MDF transport equation, Eq. (4), leads to a simple algebraic model for the scalar flux. When velocity is included in the PDF description, the transport equation for the joint velocity-scalar MDF $\mathcal{F}_{U \phi}$, Eq. (5), is modelled and solved using a particle method. In this case, the combination of the model $a_{i}$ for particle velocity evolution and the mixing model $\theta_{\alpha}$ implies a modelled transport equation for the scalar flux (and for higher order velocity-scalar correlation).

In the following, we compare results of both scalar PDF and velocity-scalar PDF calculations, using hybrid Finite-Volume / particle methods implemented in the same in-house computer program 'PDFD' [7] with the same turbulence, chemistry and mixing models. The flame considered is the Sydney bluff-body stabilised flame HM1 $[8,9,10]$ which is a target flame of the International Workshop on Measurement and Computation of Turbulent Nonpremixed Flames [11].

\section{PDF approach}

\subsection{Statistical description}

The statistical description of the flow is made in terms of the joint one-point $\operatorname{PDF} f_{\Phi}$ such that $f_{\Phi}(\boldsymbol{\Psi} ; \boldsymbol{x}, t) . \mathrm{d} \boldsymbol{\Psi}$ is the probability that $\boldsymbol{\Phi}$ is in the interval $[\boldsymbol{\Psi}, \boldsymbol{\Psi}+\mathrm{d} \boldsymbol{\Psi}]$ at point $M(\boldsymbol{x}, t)$. When the joint scalar PDF is considered, $\boldsymbol{\Phi}$ is the composition vector $\phi$. When the joint velocityscalar PDF is considered, $\boldsymbol{\Phi}=(\boldsymbol{U}, \boldsymbol{\phi})$, with $\boldsymbol{U}$ the velocity vector. The joint PDF is defined as $[1,12]$ :

$$
f_{\Phi}(\boldsymbol{\Psi} ; \boldsymbol{x}, t)=\langle\delta[\boldsymbol{\Phi}(\boldsymbol{x}, t)-\boldsymbol{\Psi}]\rangle,
$$

where $\delta[]$ is the Dirac delta function and where the brackets \langle\rangle refer to the expected value [12]. Using the conditional expected value $[1],\langle Q(\boldsymbol{x}, t) \mid \boldsymbol{\Psi}\rangle f_{\Phi}(\boldsymbol{\Psi} ; \boldsymbol{x}, t)=\langle Q(\boldsymbol{x}, t) . \delta[\boldsymbol{\Phi}(\boldsymbol{x}, t)-\boldsymbol{\Psi}]\rangle$, mean values (or expected values) are defined as:

$$
\langle Q(\boldsymbol{x}, t)\rangle=\int_{[\boldsymbol{\Psi}]}\langle Q(\boldsymbol{x}, t) \mid \boldsymbol{\Psi}\rangle f_{\Phi}(\boldsymbol{\Psi} ; \boldsymbol{x}, t) . \mathrm{d} \boldsymbol{\Psi} .
$$

Fluctuations are defined as: $\quad q^{\prime}(\boldsymbol{x}, t)=Q(\boldsymbol{x}, t)-\langle Q(\boldsymbol{x}, t)\rangle$.

For variable density flows, it is useful to consider the joint mass density function (MDF) $\mathcal{F}_{\Phi}(\boldsymbol{\Psi})=\rho(\boldsymbol{\Psi}) f_{\Phi}(\boldsymbol{\Psi})$. Density weighted averages (Favre averages) can be considered:

$$
\widetilde{Q}(\boldsymbol{x}, t)=\frac{\langle\rho(\boldsymbol{x}, t) Q(\boldsymbol{x}, t)\rangle}{\langle\rho(\boldsymbol{x}, t)\rangle}=\frac{\int_{[\boldsymbol{\Psi}]}\langle Q(\boldsymbol{x}, t) \mid \boldsymbol{\Psi}\rangle \mathcal{F}_{\Phi}(\boldsymbol{\Psi} ; \boldsymbol{x}, t) . \mathrm{d} \boldsymbol{\Psi}}{\int_{[\boldsymbol{\Psi}]} \mathcal{F}_{\Phi}(\boldsymbol{\Psi} ; \boldsymbol{x}, t) \cdot \mathrm{d} \boldsymbol{\Psi}} .
$$

Fluctuations with respect to the Favre average are defined as: $\quad q^{\prime \prime}(\boldsymbol{x}, t)=Q(\boldsymbol{x}, t)-\widetilde{Q}(\boldsymbol{x}, t)$. 


\subsection{Scalar PDF transport equation}

When the joint scalar MDF $\mathcal{F}_{\phi}$ is considered, the following transport equation is modelled and solved [1]:

$$
\frac{\partial \mathcal{F}_{\phi}}{\partial t}+\frac{\partial \widetilde{U}_{j} \mathcal{F}_{\phi}}{\partial x_{j}}+\frac{\partial}{\partial \psi_{\alpha}}\left[S_{\alpha}(\boldsymbol{\psi}) \mathcal{F}_{\phi}\right]=-\underbrace{\frac{\partial}{\partial x_{i}}\left[\left\langle u_{i}^{\prime \prime} \mid \boldsymbol{\psi}\right\rangle \mathcal{F}_{\phi}\right]}_{\text {gradient diffusion }}-\frac{\partial}{\partial \psi_{\alpha}}[\underbrace{\frac{1}{\rho(\boldsymbol{\psi})}\left\langle-\frac{\partial J_{j}^{\alpha}}{\partial x_{j}} \mid \boldsymbol{\psi}\right\rangle}_{\text {mixing model: } \theta_{\alpha}} \mathcal{F}_{\phi}]
$$

where $S_{\alpha}$ is the reaction source term for scalar $\phi_{\alpha}$ and $\boldsymbol{J}^{\alpha}$ its molecular flux.

\subsection{Velocity-scalar PDF transport equation}

When velocity is included in the PDF description, the transport equation for the joint velocityscalar MDF $\mathcal{F}_{U \phi}$ can be written (neglecting the mean viscous stress tensor gradient $\partial\left\langle\tau_{i j}\right\rangle / \partial x_{j}$ ):

$$
\begin{aligned}
\frac{\partial \mathcal{F}_{U \phi}}{\partial t}+ & V_{j} \frac{\partial \mathcal{F}_{U \phi}}{\partial x_{j}}+\left(-\frac{1}{\langle\rho\rangle} \frac{\partial\langle p\rangle}{\partial x_{i}}+g_{i}\right) \frac{\partial \mathcal{F}_{U \phi}}{\partial V_{i}}+\frac{\partial}{\partial \psi_{\alpha}}\left[S_{\alpha}(\boldsymbol{\psi}) \mathcal{F}_{U \phi}\right] \\
= & -\frac{\partial}{\partial V_{i}}[\underbrace{\left[\left(\frac{1}{\langle\rho\rangle}-\frac{1}{\rho(\boldsymbol{\psi})}\right) \frac{\partial\langle p\rangle}{\partial x_{i}}+\frac{1}{\rho(\boldsymbol{\psi})}\left\langle-\frac{\partial p^{\prime}}{\partial x_{i}}+\frac{\partial \tau_{i j}^{\prime}}{\partial x_{j}} \mid \boldsymbol{V}, \boldsymbol{\psi}\right\rangle\right]}_{\text {Langevin model: } a_{i}} \mathcal{F}_{U \phi}] \\
& -\frac{\partial}{\partial \psi_{\alpha}}[\underbrace{\left[\frac{1}{\rho(\boldsymbol{\psi})}\left\langle-\frac{\partial J_{j}^{\alpha}}{\partial x_{j}} \mid \boldsymbol{V}, \boldsymbol{\psi}\right\rangle\right.}_{\text {mixing model: } \theta_{\alpha}} \mathcal{F}_{U \phi}] .
\end{aligned}
$$

The terms on the left hand side of Eq. (5) appear in closed form. Compared to Eq. (4), effects of convection and mean pressure gradient are now exactly accounted for.

\section{Hybrid Finite-Volume / particle method}

Equations (4) and (5) are solved using the consistent hybrid Finite-Volume / particle method presented in [7].

\subsection{Finite-volume method}

Mean velocity $\widetilde{\boldsymbol{U}}$, mean pressure gradient $\nabla\langle p\rangle$, Reynolds stresses $\widetilde{u_{i}^{\prime \prime} u_{j}^{\prime \prime}}$ and turbulent dissipation $\epsilon$ are solved using a standard Finite-Volume (FV) method based on a pressure-correction algorithm.

\subsection{Particle method}

A set of uniformly distributed computational particles evolves according to stochastic differential equations. Each particle has a set of properties $\left\{w^{*}, m^{*}, \boldsymbol{X}^{*}, \boldsymbol{\phi}^{*}\right\}$ (scalar MDF), or $\left\{w^{*}, m^{*}, \boldsymbol{X}^{*}, \boldsymbol{u}^{*}, \boldsymbol{\phi}^{*}\right\}$ (velocity-scalar MDF), where $w^{*}$ is a numerical weight, $m^{*}$ is the mass of the particle, $\boldsymbol{X}^{*}$ its position, $\boldsymbol{u}^{*}$ its fluctuating velocity and $\phi^{*}$ the particle's composition. The superscript $*$ denotes that the quantity is a stochastic particle property. Particle mass $m^{*}$ is constant in time. The particle joint scalar MDF is defined as:

$$
\mathcal{F}_{\phi}^{P}(\boldsymbol{x}, \boldsymbol{\psi} ; t)=\left\langle\sum_{*} w^{*} m^{*} . \delta\left(\boldsymbol{X}^{*}(t)-\boldsymbol{x}\right) . \delta\left(\phi^{*}(t)-\boldsymbol{\psi}\right)\right\rangle .
$$


Extra properties can be deduced for each stochastic particle from the primary properties listed above. For instance, the particle density is obtained as: $\rho^{*}(t)=\rho\left[\boldsymbol{\phi}^{*}(t)\right]$.

Increments of particle position $\boldsymbol{X}^{*}$ and composition $\phi^{*}$ over small time steps $d t$ are given by:

$$
\begin{gathered}
d X_{i}^{*}=\left(U_{i}^{*}+\left[U_{i}^{c}\right]^{*}\right) d t, \\
d \phi_{\alpha}^{*}=\theta_{\alpha}^{*} d t+S_{\alpha}\left(\phi^{*}\right) d t,
\end{gathered}
$$

where the correction velocity $\boldsymbol{U}^{c}$ results from a position correction algorithm [13] which ensures that the volume represented by the particles in a computational cell equals the geometric volume of the cell. When the scalar MDF is solved, $\boldsymbol{U}^{*}$ results from a random walk model:

$$
U_{i}^{*} d t=\left[\widetilde{U}_{i}+\frac{1}{\langle\rho\rangle} \frac{\partial \Gamma_{T}}{\partial x_{i}}\right]^{*} d t+\left[\left(\frac{2 \Gamma_{T}}{\langle\rho\rangle}\right)^{1 / 2}\right]^{*} . d W_{i}^{*},
$$

where $\Gamma_{T}$ is the turbulent diffusivity ${ }^{1}$ and where $d W_{i}^{*}$ is an increment over $d t$ of the Wiener process $W_{i}^{*}$. When the joint velocity-scalar MDF is solved:

$$
\begin{aligned}
U_{i}^{*} & =\left[\widetilde{U}_{i}\right]^{*}+u_{i}^{*} \\
d u_{i}^{*} & =-u_{j}^{*}\left[\frac{\partial \widetilde{U}_{i}}{\partial x_{j}}\right]^{*} d t+\left[\frac{1}{\langle\rho\rangle} \frac{\partial\langle\rho\rangle \widetilde{u_{i}^{\prime \prime} u_{j}^{\prime \prime}}}{\partial x_{j}}\right]^{*} d t+a_{i}^{*} d t .
\end{aligned}
$$

In the above equations, the quantities between brackets [ ] ${ }^{*}$ are FV properties interpolated at the particle location using the bilinear basis functions presented in [14].

The method of fractional steps [1] is used to integrate the above systems of equations. In order to ensure second-order accuracy, the 'midpoint rule' is used [13, 14]. A local time-stepping algorithm developed in the framework of statistically stationary problems [15] is applied.

\subsection{Consistency and coupling}

Turbulent dissipation is not included in the PDF representation. The transport equation solved for $\epsilon$ in the FV method provides extra information required to model the unclosed terms $a_{i}$ and $\theta_{\alpha}$. The other FV equations are consistent with the modelled MDF transport equation [7].

The mean density $\langle\rho\rangle$ in the FV method is directly obtained from the iteration averaged mean density in the particle method ${ }^{2}$ (the iteration averaging procedure presented in [7] is used).

An outer iteration consists of a number of FV iterations and particle time steps. We use a fixed number of particle time steps (typically 5), while the FV method is iterated until the residuals of all equations start decreasing and the global mean pressure correction is below a specified threshold (with a maximum of $1000 \mathrm{FV}$ iterations per outer iteration).

\section{Modelling}

\subsection{Turbulence model}

In the context of RANS, turbulence is modelled using a second-moment closure. From the comparative study presented in [16], the isotropisation of production model by Launder, Reece and Rodi (LRR-IPM) [17] is used with the modified constant value $C_{\epsilon 1}=1.6$, instead of the standard value $C_{\epsilon 1}=1.44$. Consistently, the Lagrangian isotropisation of production model (LIPM) is used in the velocity-scalar PDF approach to describe velocity evolution $a_{i}[18,19]$.

\footnotetext{
${ }^{1} \Gamma_{T}=\mu_{T} / \mathrm{Sc}_{T}$, with $\mu_{T}$ the eddy viscosity and the turbulent Schmidt number taken as $\mathrm{Sc}_{T}=0.7$

${ }^{2}$ The global convergence of the method is improved compared to [7] where a density relaxation was used.
} 


\subsection{Chemistry model}

For the treatment of chemical reaction, the intrinsic low-dimensional manifold (ILDM) method is used to reduce the number of degrees of freedom compared to detailed reaction mechanisms [20]. Fuel (50\% $\mathrm{CH}_{4}$ and $50 \% \mathrm{H}_{2}$ by volume) and air are assumed to react in a two-stream, adiabatic system with equal diffusivities and unity Lewis number (both streams at atmospheric pressure and at a temperature of $298 \mathrm{~K}$ ). The final reduced chemistry is parametrised by three control variables: mixture fraction $(\xi)$, and $\mathrm{CO}_{2}$ and $\mathrm{H}_{2} \mathrm{O}$ mass fractions $\left(\mathrm{Y}_{\mathrm{CO}_{2}}\right.$ and $\left.\mathrm{Y}_{\mathrm{H}_{2} \mathrm{O}}\right)$. The mixture fraction $\xi$ is defined based on Bilger's Formula [21]:

$$
\xi=\frac{\frac{2\left(Z_{\mathrm{C}}-Z_{\mathrm{C}, \mathrm{o}}\right)}{W_{\mathrm{C}}}+\frac{Z_{\mathrm{H}}-Z_{\mathrm{H}, \mathrm{o}}}{2 W_{\mathrm{H}}}-\frac{Z_{\mathrm{O}}-Z_{\mathrm{O}, \mathrm{o}}}{W_{\mathrm{O}}}}{\frac{2\left(Z_{\mathrm{C}, \mathrm{f}}-Z_{\mathrm{C}, \mathrm{o}}\right)}{W_{\mathrm{C}}}+\frac{Z_{\mathrm{H}, \mathrm{f}}-Z_{\mathrm{H}, \mathrm{o}}}{2 W_{\mathrm{H}}}-\frac{Z_{\mathrm{O}, \mathrm{f}}-Z_{\mathrm{O}, \mathrm{o}}}{W_{\mathrm{O}}}} .
$$

where $Z_{\alpha}$ is the total mass fraction of element $\alpha$ (conserved scalar) and $W_{\alpha}$ is the atomic mass of element $\alpha$. The subscripts "f" and "o" refer to the fuel and oxidant streams.

In equations (4) and (5), $\phi=\left(\xi, Y_{\mathrm{CO}_{2}}, Y_{\mathrm{H}_{2} \mathrm{O}}\right)$, and the chemical source terms $S_{\mathrm{CO}_{2}}(\phi)$ and $S_{\mathrm{H}_{2} \mathrm{O}}(\phi)$ are given by the ILDM reduced chemistry.

\subsection{Mixing model}

As mixing model $\theta_{\alpha}$, the modified Curl coalescence dispersion (CD) model is used. The CD micromixing model prescribes the evolution of particle composition as a series of pairwise mixing events. The particles participating in mixing are chosen at random from the set of particles present in a finite volume cell and their compositions change in the direction of the partner. The degree of mixing in a pair is determined by a random variable uniformly distributed between 0 (no mixing) and 1 (complete mixing) [22, 23].

\subsection{Implied modelled equations for mean scalar, scalar variance and scalar flux}

From Eq. (4) or (5), we formally obtain the same mean scalar transport equation and scalar variance equation:

$$
\begin{gathered}
\frac{\partial\langle\rho\rangle \widetilde{\phi_{\alpha}}}{\partial t}+\frac{\partial\langle\rho\rangle \widetilde{U_{j}} \widetilde{\phi_{\alpha}}}{\partial x_{j}}=-\frac{\partial\langle\rho\rangle \widetilde{u_{j}^{\prime \prime} \phi_{\alpha}^{\prime \prime}}}{\partial x_{j}}+\langle\rho\rangle \widetilde{S_{\alpha}}+\underbrace{\left\langle\rho \theta_{\alpha}\right\rangle}_{=0 \text { (mixing model property) }} \\
\frac{\partial\langle\rho\rangle \widetilde{\phi_{\alpha}^{\prime \prime 2}}}{\partial t}+\frac{\partial\langle\rho\rangle \widetilde{U_{j}} \widetilde{\phi_{\alpha}^{\prime \prime 2}}}{\partial x_{j}}+2\langle\rho\rangle \widetilde{u_{j}^{\prime \prime} \phi_{\alpha}^{\prime \prime}} \frac{\partial \widetilde{\phi_{\alpha}}}{\partial x_{j}}=-\frac{\partial\left\langle\rho u_{j}^{\prime \prime} \phi_{\alpha}^{\prime \prime 2}\right\rangle}{\partial x_{j}}-2\langle\rho\rangle \widetilde{\phi_{\alpha}^{\prime \prime} S_{\alpha}}-2\left\langle\rho \phi_{\alpha}^{\prime \prime} \theta_{\alpha}\right\rangle,
\end{gathered}
$$

with no implicit summation on $\alpha$. In Eq. (14), the modelling of the last term (scalar dissipation and molecular diffusion) results from the mixing model $\theta_{\alpha}$ and is the same in both PDF approaches.

When the modelled joint scalar MDF is solved, the gradient diffusion assumption (random walk model in the particle method) implies the following algebraic models for velocity-scalar correlation:

$$
\left\langle\rho u_{j}^{\prime \prime} \phi_{\alpha}^{\prime \prime}\right\rangle=-\Gamma_{T} \frac{\partial \widetilde{\phi_{\alpha}}}{\partial x_{j}} \quad \text { and } \quad\left\langle\rho u_{j}^{\prime \prime} \phi_{\alpha}^{\prime 2}\right\rangle=-\Gamma_{T} \frac{\partial \widetilde{\phi_{\alpha}^{\prime \prime 2}}}{\partial x_{j}}
$$

When the joint velocity-scalar MDF is considered, a differential scalar flux model is actually implied, depending on the Langevin model $a_{i}$ and the mixing model $\theta_{\alpha}$. For instance, the 
modelled scalar flux transport equation takes the form:

$$
\begin{aligned}
\frac{\partial\langle\rho\rangle \widetilde{u_{i}^{\prime \prime} \phi_{\alpha}^{\prime \prime}}}{\partial t} & +\frac{\partial\langle\rho\rangle \widetilde{U}_{j} \widetilde{u_{i}^{\prime \prime} \phi_{\alpha}^{\prime \prime}}}{\partial x_{j}}+\langle\rho\rangle \widetilde{u_{j}^{\prime \prime} \phi_{\alpha}^{\prime \prime}} \frac{\partial \widetilde{U}_{i}}{\partial x_{j}}+\langle\rho\rangle \widetilde{u_{i}^{\prime \prime} u_{j}^{\prime \prime}} \frac{\partial \widetilde{\phi}_{\alpha}}{\partial x_{j}} \\
& =-\frac{\left\langle\rho u_{j}^{\prime \prime} u_{i}^{\prime \prime} \phi_{\alpha}^{\prime \prime}\right\rangle}{\partial x_{j}}+\langle\rho\rangle \widetilde{u_{i}^{\prime \prime} S_{\alpha}}+\left\langle\rho a_{i} \phi_{\alpha}^{\prime \prime}\right\rangle+\left\langle\rho u_{i}^{\prime \prime} \theta_{\alpha}\right\rangle .
\end{aligned}
$$

\section{Test case description}

\subsection{Bluff-body stabilised flame HM1}

The fuel (50\% $\mathrm{H}_{2}$ and $50 \% \mathrm{CH}_{4}$ by volume) is injected in the centre of the bluff-body burner through an injector of diameter $D_{j}=3.6 \mathrm{~mm}$. The bluff body of diameter $D_{b}=50 \mathrm{~mm}$ is surrounded by an unconfined coflowing air stream. Fuel and air are mixed in the recirculation zone behind the bluff body where chemical reaction can occur. The resulting hot products stabilise the flame.

In the experimental studies [8] and [9], the jet and coflow bulk velocities were respectively $118 \mathrm{~m} / \mathrm{s}$ and $40 \mathrm{~m} / \mathrm{s}$ (flame HM1). More recently, two sets of velocity measurements were provided by Kalt and Masri [10] for reduced jet and coflow bulk velocities of $108 \mathrm{~m} / \mathrm{s}$ and $35 \mathrm{~m} / \mathrm{s}$ (flame HM1e).

\subsection{Numerical settings}

The numerical settings are similar to the calculations of flame HM1e presented in [7]. Note that in the present study flame HM1 is considered.

A $6 D_{b}$-long and $3 D_{b}$-wide $2 \mathrm{D}$ computational domain is used. Free-slip boundary conditions are prescribed on the bluff-body surface and on the lateral boundary. A convective outlet boundary condition [24] is used in order to avoid reflecting waves. Inlet boundary conditions are specified at cell centres in the same way as done in [25]. Results are obtained on a $160 \times 128$ cartesian grid stretched both in axial and radial directions.
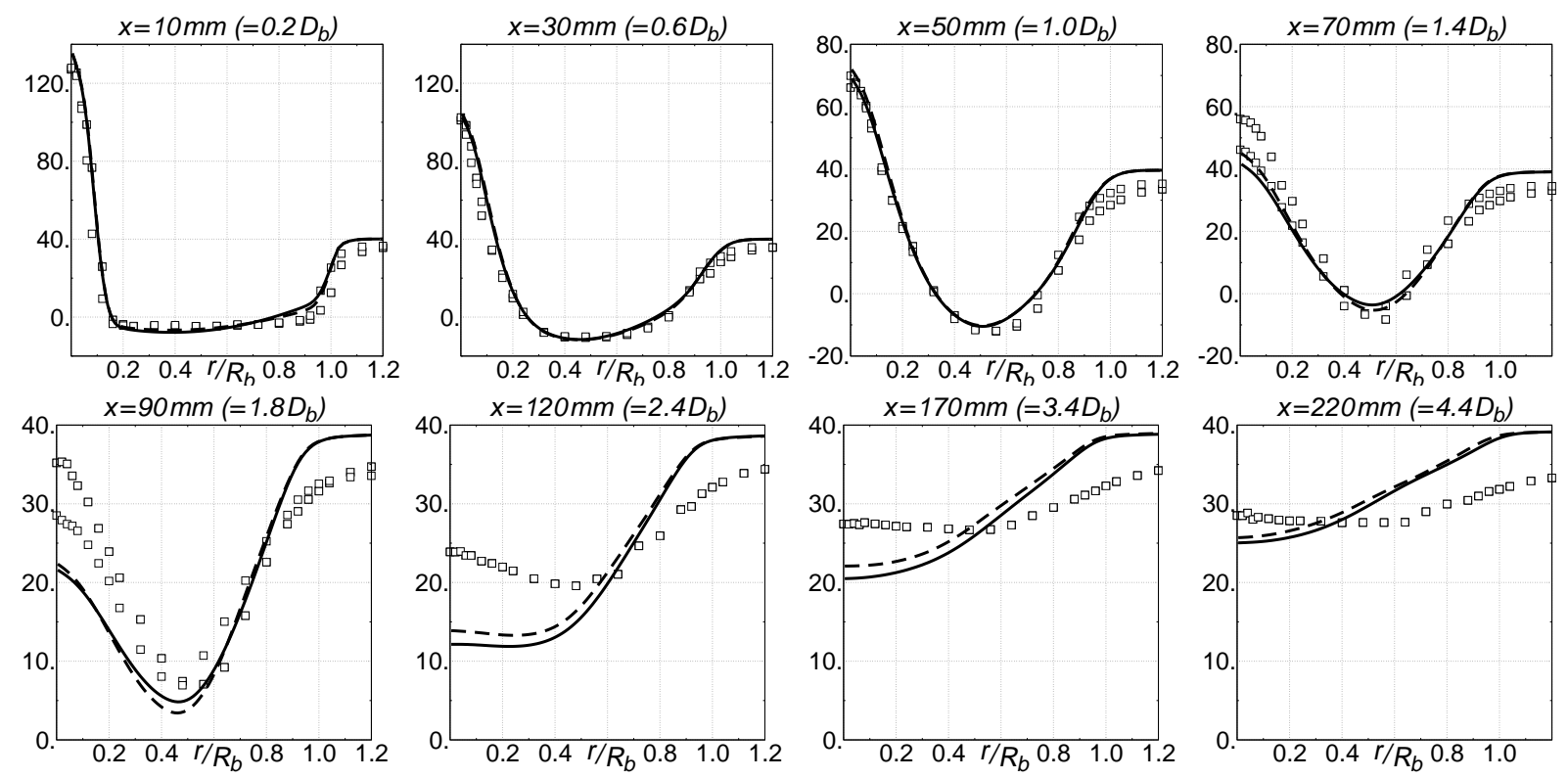

Figure 1: Radial profiles of mean axial velocity $\widetilde{U}$. Symbols: measurements in flame HM1e (two sets of measurements). Solid line: scalar PDF results. Dashed line: velocity-scalar PDF results. 

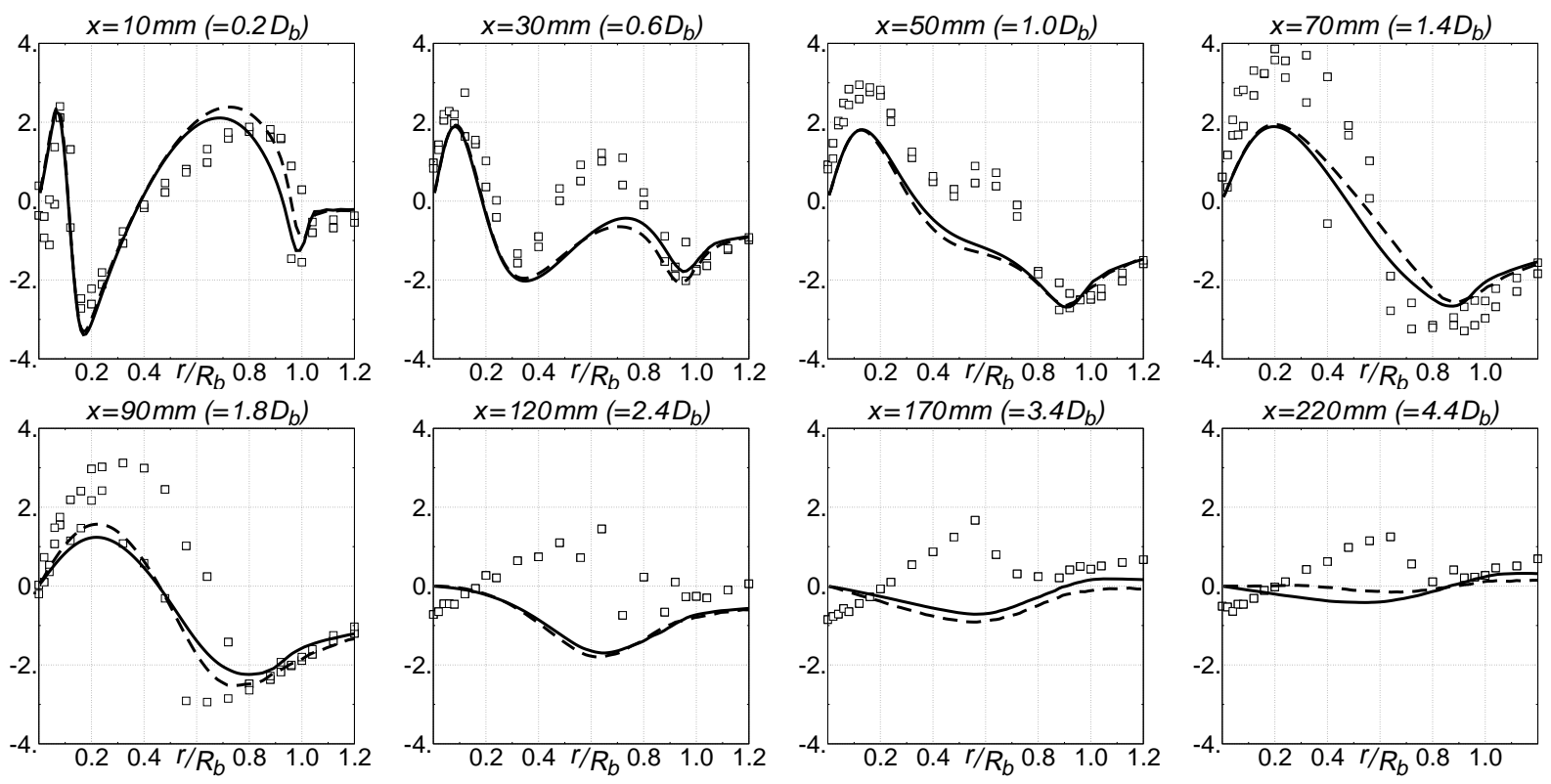

Figure 2: Radial profiles of mean radial velocity $\widetilde{V}$. Symbols: measurements in flame HM1e (two sets of measurements). Solid line: scalar PDF results. Dashed line: velocity-scalar PDF results.
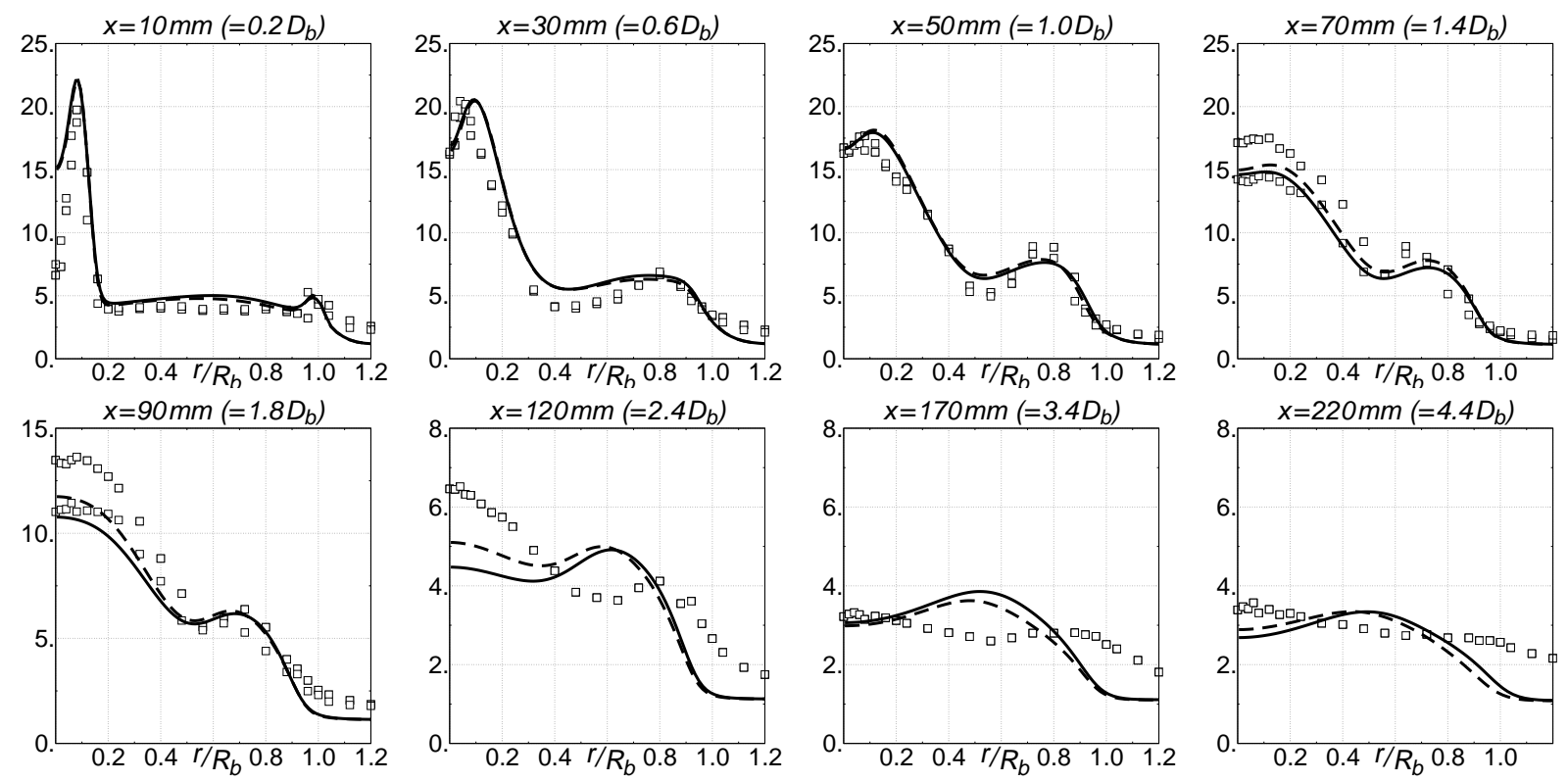

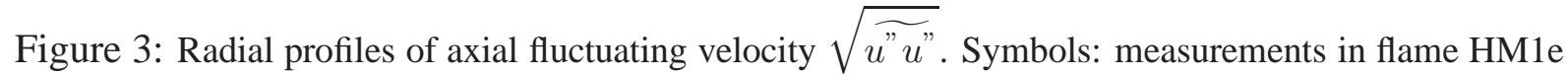
(two sets of measurements). Solid line: scalar PDF results. Dashed line: velocity-scalar PDF results.

An average of 100 particles per cell is used. Iteration averages are made over 500 iterations. The coupling between FV and particle methods is done as described in 3.3. As in [7], converged results obtained using an assumed-shape PDF method are used as initial conditions. About 1000 outer iterations (5000 particle time steps) are enough to reach a stationary solution and take typically one day of calculation time on a single processor 'Dual Xeon $2.4 \mathrm{GHz}$ '. Results obtained after 15000 particle time steps are now discussed. 


\section{Results}

\subsection{Mean velocity and Reynolds stresses}

Our velocity results for flame HM1 are compared to the velocity measurements from flame HM1e. The results shown in Fig. 1, 2 and 3 for mean axial velocity, mean radial velocity and fluctuating axial velocity are very similar to the results of [7] or [25, 26]. As discussed in [26], good agreement with experimental data is observed within the recirculation zone. The agreement deteriorates in the neck zone and the jet-like zone (from about $x=1.8 D_{b}$ ). These downstream discrepancies have been observed in the different RANS calculations presented at the TNF workshops [11].

An important observation for the present study is that the very small differences between scalar PDF and velocity-scalar PDF results (due to some differences in mean density) are negligible.

\subsection{Mean composition}
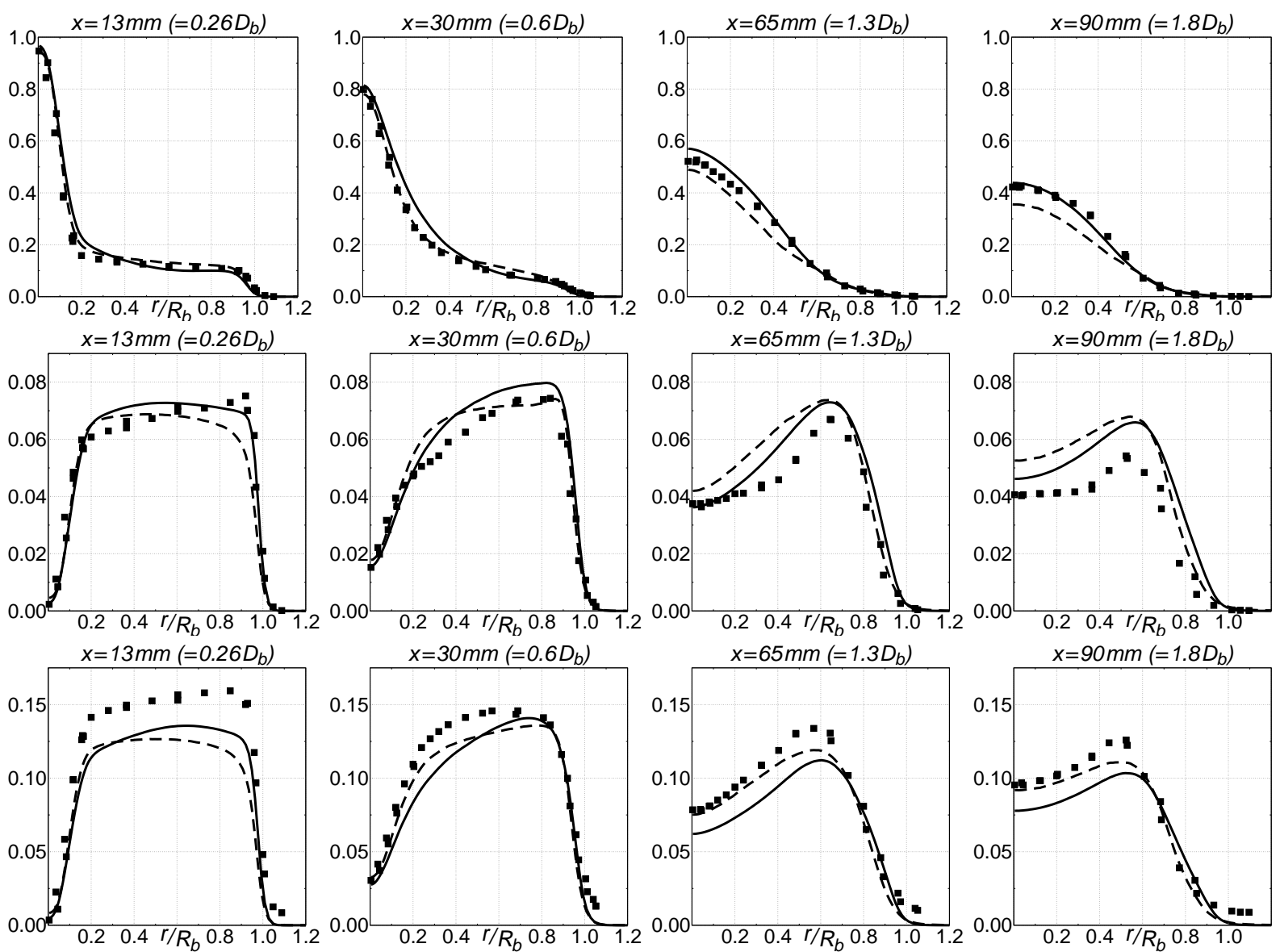

Figure 4: Radial profiles of mean mixture fraction $\widetilde{\xi}$ (up), $\mathrm{CO}_{2}$ mass fraction $\widetilde{Y_{C O_{2}}}$ (middle) and $\mathrm{H}_{2} \mathrm{O}$ mass fraction $\widetilde{\mathrm{YH}_{2} \mathrm{O}}$ (down). Symbols: measurements in flame HM1. Solid line: scalar PDF results. Dashed line: velocity-scalar PDF results.

Fig. 4 shows mean radial profiles for the three scalars $\xi, Y_{\mathrm{CO}_{2}}$ and $Y_{\mathrm{H}_{2} \mathrm{O}}$. Significant differences between scalar PDF and velocity-scalar PDF results are observed. It is not clear which results are the best for $Y_{\mathrm{CO}_{2}}$ and $Y_{\mathrm{H}_{2} \mathrm{O}}$. For mean mixture fraction, best results are obtained with velocity-scalar PDF. They are better than previously reported joint scalar-velocity(-frequency) 
results $[7,25]$. Looking at the mean transport equation, Eq. (13), helps to understand the observed differences. We can neglect the influence of mean density, as suggested by mean velocity and Reynolds stress results. Hence, for mixture fraction (no reaction source term), the difference is due to the different modelling of the scalar flux $\widetilde{u_{j}^{\prime \prime} \xi^{\prime \prime}}$.

The relative differences observed for $Y_{\mathrm{CO}_{2}}$ and $Y_{\mathrm{H}_{2} \mathrm{O}}$ are similar to those observed for $\xi$ for similar mean gradients (with opposite sign). This suggests that scalar flux modelling is also a major source of difference for the reacting scalars.

\subsection{Mixture fraction variance}

In Fig. 5, differences are also observed for radial profiles of mixture fraction variance $\widetilde{\xi^{\prime \prime 2}}$. In general, velocity-scalar PDF results are in better correspondence with the experimental data. The observed differences are related to the modelling of velocity-scalar correlation: the scalar flux $\widetilde{u_{j}^{\prime \prime} \xi^{\prime \prime}}$ appearing in the production term and the triple correlation $u_{j}^{\prime \prime} \xi^{\prime \prime} \xi^{\prime \prime}$ appearing in the turbulent diffusion term in Eq. (14).
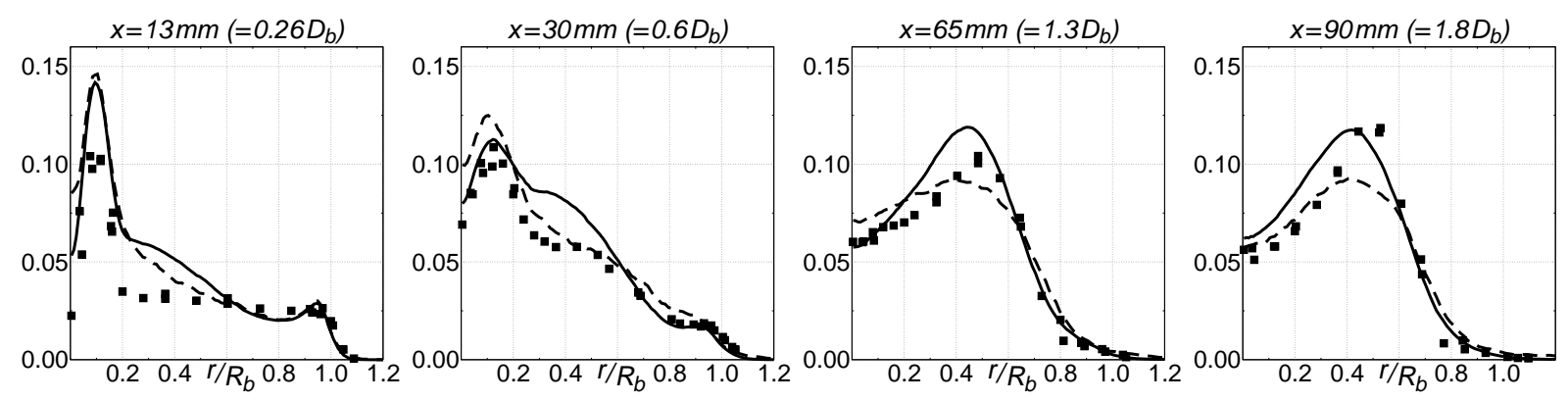

Figure 5: Radial profiles of mixture fraction fluctuation $(\mathrm{rms}) \sqrt{\widetilde{\xi^{\prime \prime}}}$. Symbols: measurements in flame HM1. Solid line: scalar PDF results. Dashed line: velocity-scalar PDF results.

\subsection{Results in composition space: scatter plots}

The above observations on the impact of the choice of the PDF method on mean scalar profiles is not surprising. The higher quality of a differential scalar flux model compared to a gradient diffusion assumption was to be expected, especially in a flow with a strong recirculation zone. We now focus on the impact of the choice of the PDF method on the predicted joint PDF of $\xi$ and $Y_{\mathrm{CO}_{2}}$ (similar observations can be made on the joint PDF of $\xi$ and $Y_{\mathrm{H}_{2} \mathrm{O}}$ ).
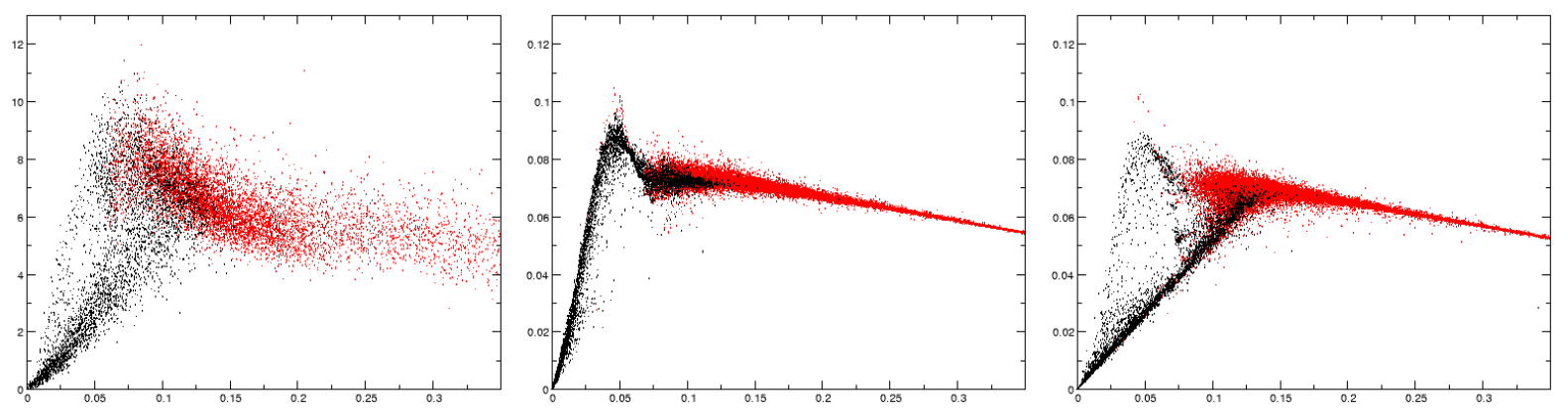

Figure 6: Scatter plots of $Y_{\mathrm{CO}_{2}}$. First measurement section $(x=13 \mathrm{~mm})$. Black: $r \in[23 \mathrm{~mm} ; 25 \mathrm{~mm}]$. Red: $r<23 \mathrm{~mm}$. Left: experimental data (in percent). Middle: scalar PDF. Right: velocity-scalar PDF. 
The scatter plots of $Y_{\mathrm{CO}_{2}}$ shown in Fig. 6 show the strong impact of the choice of PDF method on the predicted joint PDF of mixture fraction and $\mathrm{CO}_{2}$ at the radial cross section $x=13 \mathrm{~mm}$. For the considered CD mixing model, at the lean side, the scalar PDF shows remarkably more particles at fully burnt conditions than the velocity-scalar PDF, with the latter being in better qualitative agreement with the experiment.

This trend is still observed in Fig. 7 at $x=30 \mathrm{~mm}$. However, Fig. 8 shows that further downstream, at $x=65 \mathrm{~mm}$, scalar PDF and velocity-scalar PDF methods lead to similar shapes of the joint PDF. The joint scalar PDF study of [4] suggests that this could be related to the tendency of the CD mixing model towards uniform conditional fluctuation intensity downstream of the recirculation region. Further studies will be needed in order to clarify this point.
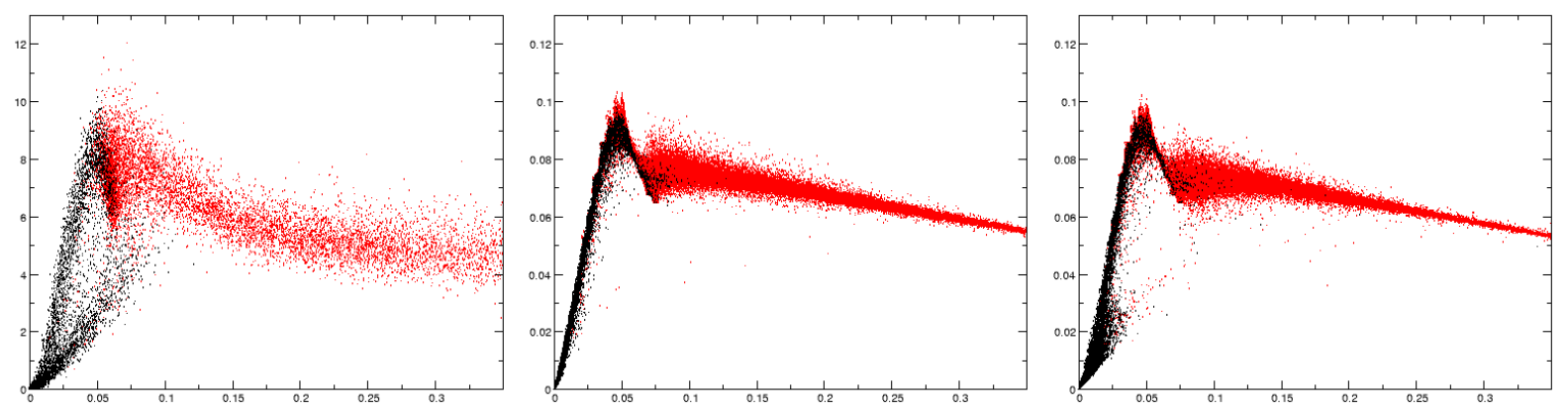

Figure 7: Scatter plots of $Y_{\mathrm{CO}_{2}}$ at $x=30 \mathrm{~mm}$. Black: $r \in$ [23mm;25mm]. Red: $r<23 \mathrm{~mm}$. Left: experimental data (in percent). Middle: scalar PDF. Right: velocity-scalar PDF.
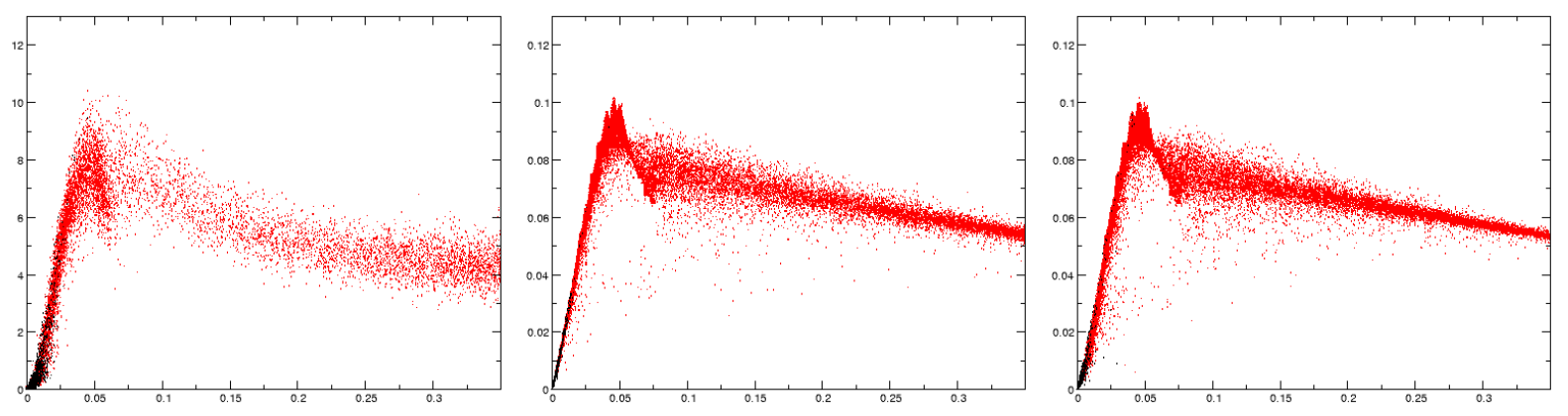

Figure 8: Scatter plots of $Y_{\mathrm{CO}_{2}}$ at $x=65 \mathrm{~mm}$. Black: $r \in$ [23mm;25mm]. Red: $r<23 \mathrm{~mm}$. Left: experimental data (in percent). Middle: scalar PDF. Right: velocity-scalar PDF.

As a final remark, we note that the level of scatter in the present results, obtained with the CD mixing model, is in better correspondence with the experimental data than in the results of [26] obtained with the EMST mixing model. This is actually the reason why the CD mixing model was used in the present study.

\section{Conclusions}

A fair comparison of scalar PDF and velocity-scalar PDF modelling of the bluff-body flame HM1 has been conducted. Differences in the mean flow are negligible. Significant differences are observed in results for mean scalars and mixture fraction variance. Not surprisingly, velocity-scalar PDF results (implying a differential scalar flux model) are in general better than scalar PDF results (based on gradient diffusion assumption). Results in composition space give more direct information on how effects of mixing and reaction combine. With the CD mixing 
model used in this study, best qualitative agreement of scatter plots in the near field $(x=13 \mathrm{~mm})$ are obtained with the velocity-scalar PDF.

\section{Acknowledgements}

The second author is Postdoctoral Fellow of the Fund of Scientific Research - Flanders (Belgium) (FWO-Vlaanderen). This collaborative research is supported by the Spanish MEC under Project \#ENE2005-09190-C04-04/CON and by the Fund of Scientific Research - Flanders (Belgium) (FWO-Vlaanderen) through FWO-project G.0070.03.

\section{References}

1. S.B. Pope. PDF methods for turbulent reactive flows. Progress in Energy and Combustion Science, 11:119-192, 1985.

2. Z. Ren and S.B. Pope. An investigation of the performance of turbulent mixing models. Combustion and Flame, 136:208-216, 2004.

3. B. Merci, D. Roekaerts and B. Naud. Study of the performance of three micromixing models in transported scalar PDF simulations of a piloted jet diffusion flame ("Delft Flame III"). Combustion and Flame, 144:476-493, 2006.

4. B. Merci, D. Roekaerts, B. Naud and S.B. Pope. Comparative Study of Micro-Mixing Models in Transported Scalar PDF Simulations of Turbulent Non-Premixed Bluff Body Flames. Combustion and Flame, http://doi:10.1016/j.combustflame.2006.04.010

5. R.R. Cao and S.B. Pope. The influence of chemical mechanisms on PDF calculations of nonpremixed piloted jet flames. Combustion and Flame, 143:450-470, 2005.

6. B. Merci, B. Naud and D. Roekaerts. Interaction between chemistry and micro-mixing modeling in transported PDF simulations of turbulent non-premixed flames. Submitted.

7. B. Naud, C. Jiménez and D. Roekaerts. A consistent hybrid PDF method: implementation details and application to the simulation of a bluff-body stabilised flame. Progress in Computational Fluid Dynamics, 6:146-157, 2006.

8. B.B. Dally and A.R. Masri. Flow and mixing fields of turbulent bluff-body jets and flames. Combustion Theory and Modelling, 2:193-219, 1998.

9. B.B. Dally, A.R. Masri, R.S. Barlow, and G.J. Fiechtner. Instantaneous and mean compositional structure of bluff-body stabilized nonpremixed flames. Combustion and Flame, 114:119-148, 1998.

10. http://www.aeromech.usyd.edu.au/thermofluids/main_frame.htm

11. R.S. Barlow. International Workshop on Measurement and Computation of Turbulent Nonpremixed Flames. http://www.ca.sandia.gov/TNF

12. S.B. Pope. Turbulent Flows, Cambridge University Press, 2000.

13. M. Muradoglu, S.B. Pope, and D.A. Caughey. The hybrid method for the PDF equations of turbulent reactive flows: consistency conditions and correction algorithms. Journal of Computational Physics, 172:841-878, 2001.

14. P. Jenny, S.B. Pope, M. Muradoglu, and D.A. Caughey. A hybrid algorithm for the joint PDF equation of turbulent reactive flows. Journal of Computational Physics, 166:218-252, 2001.

15. M. Muradoglu and S.B. Pope. A local time-stepping algorithm for solving the Probability Density Function turbulence model equations. AIAA Journal, 40:1755-1763, 2002.

16. G. Li, B. Naud and D. Roekaerts. Numerical Investigation of a Bluff-Body Stabilised Nonpremixed Flame with Differential Reynolds-Stress Models. Flow, Turbulence and Combustion, 70:211-240, 2003. 
17. B.E. Launder, G.J. Reece and W. Rodi. Progress in the development of a Reynolds-stress turbulence closure. Journal of Fluid Mechanics, 68:537-566, 1975.

18. S.B. Pope. On the relationship between stochastic Lagrangian models of turbulence and second-moment closures. Physics of Fluids, 6:973-985, 1994.

19. H.A. Wouters, T.W.J. Peeters and D. Roekaerts. On the existence of a Generalized Langevin model representation for second-moment closures. Physics of Fluids, 8:17021704, 1996.

20. U. Maas and S.B. Pope. Simplifying chemical kinetics: intrinsic low-dimensional manifolds in composition space. Combustion and Flame, 88:239-264, 1992.

21. R.W. Bilger, S.H. Starner and R.J. Kee. On reduced mechanisms for methane-air combustion in nonpremixed flames. Combustion and Flame, 80:135-149, 1990.

22. J. Janicka, W. Kolbe and W. Kollmann. Closure of the transport equation for the probability density function of turbulent scalar fields. Journal Non-Equilibrium Thermodynamics, 4:47-66, 1979.

23. H.A. Wouters, T.W.J. Peeters, and D. Roekaerts. Joint velocity-scalar PDF methods. Chapter 2 in Closure strategies for turbulent and transitional flows (Edited by B. Launder and N. Sandham), Cambridge University Press, pp. 626-655, 2002.

24. A. Sohankar, C. Norberg, and L. Davidson. Low-Reynolds-number flow around a square cylinder at incidence: study of blockage, onset of vortex shedding and outlet boundary condition. International Journal for Numerical Methods in Fluids, 26:39-56, 1998.

25. M. Muradoglu, K. Liu, and S.B. Pope. PDF modeling of a bluff-body stabilized turbulent flame. Combustion and Flame, 132:115-137, 2003.

26. K. Liu, S.B. Pope and D.A. Caughey. Calculations of bluff-body stabilized flames using a joint probability density function model with detailed chemistry. Combustion and Flame, 141:89-117, 2005. 